\title{
siRNA against plasminogen activator inhibitor-1 ameliorates bleomycin-induced lung fibrosis in rats
}

\author{
Yan-ping ZHANG ${ }^{1,2}$, Wen-bin $\mathrm{LI}^{1,4, *}$, Wei-li WANG ${ }^{1}$, Jian $\mathrm{LIU}^{2}$, Shu-xia SONG ${ }^{3}$, Lin-lin BAI ${ }^{2}$, Yu-yan $\mathrm{HU}^{1}$, Ya-dong YUAN ${ }^{2}$, Min \\ ZHANG $^{1}$ \\ ${ }^{1}$ Department of Pathophysiology, Hebei Medical University, Shijiazhuang 050017, China; ${ }^{2}$ Department of Respiration, the Second Hos- \\ pital of Hebei Medical University, Shijiazhuang 050000, China; ${ }^{3}$ Department of Immunology, Hebei Medical University, Shijiazhuang \\ 050017, China; ${ }^{4}$ Aging and Cognition Neuroscience Laboratory of Hebei Province, Shijiazhuang 050031, China
}

\begin{abstract}
Aim: Plasminogen activator inhibitor-1 (PAI-1) is involved in the progression of pulmonary fibrosis. The present study was undertaken to examine the effects on pulmonary fibrosis of silencing PAl-1 expression with small interfering RNA (siRNA) and to assess the possible underlying mechanisms.

Methods: Male Wistar rats were subjected to intratracheal injection of bleomycin (BLM, $5 \mathrm{mg} / \mathrm{kg}, 0.2 \mathrm{~mL}$ ) to induce pulmonary fibrosis. Histopathological changes of lung tissue were examined with HE or Masson's trichrome staining. The expression levels of $\alpha$-smooth muscle actin $(\alpha-S M A)$, collagen type-I and type-III, caspase-3, as well as p-ERK1/2 and PI3K/Akt in the lung tissue were evaluated using immunohistochemistry and Western blot analysis. The fibroblasts isolated from BLM-induced fibrotic lung tissue were cultured and transfected with pcDNA-PAI-1 or PAI-1 siRNA. The expression level of PAI-1 in the fibroblasts was measured using real time RT-PCR and Western blot analysis. The fibroblast proliferation was evaluated using MTT assay.

Results: Intratracheal injection of PAl-1 siRNA (7.5 nmoL/0.2 mL) significantly alleviated alveolitis and collagen deposition, reduced the expression of PAI-1, $\alpha$-SMA, collagen type-I and collagen type-III, and increased the expression of caspase-3 in BLM-induced fibrotic lung tissue. In consistence with the in vivo results, the proliferation of the cultured fibroblasts from BLM-induced fibrotic lung tissue was inhibited by transfection with PAl-1 siRNA, and accelerated by overexpression of PAl-1 by transfection with pcDNA-PAI-1. The expression of caspase- 3 was increased as a result of PAI-1 siRNA transfection, and decreased after transfection with pcDNA-PAI-1. In addition, the levels of $\mathrm{p}-\mathrm{ERK} 1 / 2$ and PI3K/Akt in the fibrogenic lung tissue were reduced after treatment with PAI-1 siRNA.

Conclusion: The data demonstrate that PAI-1 siRNA inhibits alveolitis and pulmonary fibrosis in BLM-treated rats via inhibiting the proliferation and promoting the apoptosis of fibroblasts. Suppression ERK and AKT signalling pathways might have at least partly contributed to this process. Targeting PAI-1 is a promising therapeutic strategy for pulmonary fibrosis.
\end{abstract}

Keywords: idiopathic pulmonary fibrosis; pulmonary fibroblast; bleomycin; plasminogen activator inhibitor-1; RNA interference; $\alpha$-smooth muscle actin; collagen; caspase-3; extracellular signal-regulated kinase; phosphatidylinositol 3-phosphate kinase

Acta Pharmacologica Sinica (2012) 33: 897-908; doi: 10.1038/aps.2012.39; published online 4 Jun 2012

\section{Introduction}

Idiopathic pulmonary fibrosis (IPF) is characterised by the transformation of fibroblasts into myofibroblasts and by the accumulation of excessive extracellular matrix $(\mathrm{ECM})^{[1]}$. Decreasing the deposition of ECM and enhancing its degradation might be particularly valuable in the treatment of lung fibrosis $^{[2]}$. However, specific knowledge-based approaches for the prevention and efficacious treatment of lung fibrosis are lacking. Increasing evidence supports the pivotal role of the fibrinolysis system, including urokinase-type plasminogen

\footnotetext{
* To whom correspondence should be addressed.

E-mail liwbsjz@yahoo.com.cn

Received 2012-02-27 Accepted 2012-03-23
}

activator (uPA) and its receptor, inhibitor of UPA (PAI-1), and plasminogen in regulating the balance between ECM degradation and deposition ${ }^{[3]}$. Bueno et al observed that transfection with the uPA gene could induce cirrhosis regression and ameliorate hepatic dysfunction ${ }^{[4]}$. Hattori et al revealed that the plasminogen activation system reduces lung fibrosis through a hepatocyte growth factor-dependent mechanism ${ }^{[5]}$.

The fibrin deposits persist in patients with IPF because normal fibrinolytic activity is suppressed by an increased expression of PAI- ${ }^{[6]}$. Recent evidence has shown that PAI-1 regulates proliferation and apoptosis in different cell lines by activating the ERK, AKT, JAK-STAT, and NF-KB signalling pathways. Experimental studies have also revealed the important role of PAI-1 in the pathogenesis of fibrotic disease. 
For example, Eitzman et al revealed that overexpression of PAI-1 could lead to more severe bleomycin-induced pulmonary fibrosis ${ }^{[7]}$, whereas mice with a targeted deletion of the PAI-1 gene (PAI-1 ${ }^{-/-}$mice) developed less fibrosis and survive longer ${ }^{[8]}$. Interestingly, silencing PAI-1 expression alleviated hepatic fibrosis induced by $\mathrm{CCl}_{4}{ }^{[9]}$. However, it remains unclear whether silencing PAI-1 expression could ameliorate lung fibrosis. In 2010, Senoo et al reported that PAI-1 siRNA prevented pulmonary fibrosis by inhibition of the epithelialto-mesenchymal transition $(\mathrm{EMT})^{[10]}$. However, whether PAI-1 siRNA inhibits the proliferation of fibroblasts directly and its effects on the related signalling pathways have not been determined.

Therefore, the present study was undertaken to observe whether silencing PAI-1 expression with siRNA against PAI-1 could inhibit lung fibrosis and whether the mechanisms of ameliorating fibrosis with siRNA against PAI-1 are related to the regulation of (myo)fibroblasts proliferation and apoptosis through the modulation of ERK and AKT signalling molecules, in a rat model of bleomycin (BLM)-induced pulmonary fibrosis.

\section{Materials and methods}

\section{Animals and grouping}

In accordance with to a report by $\mathrm{Hu}$ et $a l^{[9]}$, the siRNA against rat PAI-1 mRNA and non-specific siRNA (Ns-siRNA) (designed and synthesised by Guangzhou RiboBio Co, LTD) were selected for evaluating in BLM-induced pulmonary fibrosis in rats. The siRNA sequences mentioned above are shown in Table 1.

Table 1. The sequences of small interfering RNA used for plasminogen activator inhibitor-1 (PAl-1) silencing.

\begin{tabular}{lll}
\hline 559 siRNA & Sense & 5'-GGACUUCUCAGAGGUGGAATT-3' \\
& Antisense & 5'-UUCCACCUCUGAGAAGUCCTT-3' \\
Non-specific siRNA & Sense & 5'-UUCUCCGAACGUGUCACGUTT-3' \\
& Antisense & 5'-ACGUGACACGUUCGGAGAATT-3' \\
\hline
\end{tabular}

A total of 72 male Wistar rats weighing 130-140 g were provided by the Experimental Animal Center of Hebei Medical University, China. All rats were housed in the pathogen-free mosue colony and all experiments were performed according to the guidelines for the care and use of medical laboratory animals (Ministry of Health, China, 1998). The rats were divided into 4 treatment groups: sham, BLM, Non-specific siRNA (Ns-siRNA) and siRNA. The tracheas of the rats were exposed, and the rats in the BLM group were subjected to intratracheal injection of BLM $(5 \mathrm{mg} / \mathrm{kg}, 0.2 \mathrm{~mL})$ under chloral hydrate anaesthesia $(10 \%, \mathrm{ip}, 350 \mathrm{mg} / \mathrm{kg})$, whereas the rats in the sham group received an equal volume of normal saline. The rats in the siRNA and Non-specific siRNA groups were first treated with BLM according to the protocol described above. From the third day after the administration of BLM, the rats were treated with tracheal administration of PAI1-siRNA or non-specific siRNA (7.5 nmoL/0.2 mL per rat), respectively, once every three days. For all groups, on $\mathrm{d} 7,14$, and 28 after the administration of BLM or normal saline, the rats ( $n=6$ at each time point) were sacrificed and the lower lobe of the right lung was harvested for histological observation, immunostaining, real time RT-PCR and Western blot analysis. Bronchoalveolar lavage (BAL) was performed to determine the PAI-1 activity using a colorimetric assay (American Diagnostica Inc, USA) according to the manufacturer's instructions.

\section{Histological and immunohistochemical assays}

Haematoxylin-Eosin (HE) or Masson's trichrome staining were conducted to examine the histopathological changes of lung tissue. Immunohistochemical examination was carried out to detect the expression of a-SMA, collagen type-I and type-III, and caspase- 3 in the lung tissue using the following primary antibodies: rabbit anti-a-SMA (Epitomics, USA), goat anti-collagen type-I and type-III (Santa Cruz), and rabbit anticaspase-3 (Santa Cruz) antibodies.

\section{Cell isolation and culture}

The rat fibroblasts from BLM-induced fibrotic lung tissue were performed to evaluate the effect of expression of the siRNA and pcDNA-PAI-1 on proliferation and apoptosis in vitro. Freshly harvested lung tissues were cut into $1 \times 1 \times 1 \mathrm{~mm}^{3}$ strips and maintained in Dulbecco's modified Eagle's medium (DMEM) (Gibco, USA) supplemented with $100 \mathrm{U} / \mathrm{mL}$ penicillin, $100 \mu \mathrm{g} / \mathrm{mL}$ streptomycin, and 15\% heat-inactivated foetal calf serum (Gibco, USA). After being cultured for 2-3 passages, purified fibroblasts were obtained and identified by immunohistochemistry staining with antibodies against vimentin and a-SMA proteins. Early-passage cells (2-4 passages) were used in the subsequent experiments.

\section{Determination of fibroblast proliferation and apoptosis}

The effects of transfection with pcDNA-PAI-1 (provided by Dr Fang WANG) on the expression levels of PAI-1 were evaluated by Western blot analysis at $48 \mathrm{~h}$ and $72 \mathrm{~h}$, and the effects on fibroblast proliferation were evaluated using the 3-(4,5-dimethylthiazol-2-yl)-2,5-diphenyltetrazolium bromide (MTT) assay. The cells were transfected with PAI-1 siRNA or pcDNA-PAI-1 using the Lipofectamine 2000 transfection reagent (Invitrogen, Carlsbad, CA, USA) according to the manufacturer's instructions. Briefly, the cells were trypsinised and diluted in fresh culture medium without antibiotics, plated at a density of $2 \times 10^{4}$ cells/well in triplicate wells on a 96-well plate and cultured for $24 \mathrm{~h}$. The number of viable cells was determined at $570 \mathrm{~nm}$ at 24, 48, and $72 \mathrm{~h}$ after transfection with PAI-1 siRNA or pcDNA-PAI-1. Apoptosis was evaluated by detection of caspase-3 expression in the fibroblasts by real time RT-PCR at $24 \mathrm{~h}$ after transfection with PAI-1 siRNA or pcDNA-PAI-1.

Real time reverse transcription polymerase chain reaction Total RNA was extracted from the lung tissues and cultured 
Table 2. Primer sequences for real time reverse transcriptase polymerase chain reaction.

\begin{tabular}{lll}
\hline PAI-1 & Forward & 5'-CCTTCCAGAGTCCCATACA-3' \\
& Reverse & 5'-CTGG CTCTTTCCACCTCT-3' \\
Collagen type-I & Forward & 5'-GGTGGTTATGACTTCAGCTTCC-3' \\
& Reverse & 5'-CATGTAGGCTACGCTGTTCTTG-3' \\
Collagen type-III & Forward & 5'-GTCTTATCAGCCCTGATGGTTC-3' \\
& Reverse & 5'-GCTCCATTCACCAGTGTGTTTA-3' \\
Caspase-3 & Forward & 5'-AATTCAAGGGACGGGTCATG-3' \\
& Reverse & 5'-GCTTGTGCGCGTACAGTTTC-3' \\
GAPDH & Forward & 5'-CCATGTTTGTGATGGGTGTGACCA-3' \\
& Reverse & 5'-ACCAGTGGATGCAGGATGATGTTC-3' \\
\hline
\end{tabular}

fibroblasts using Trizol reagent (TaKaRa, Japan) according to the manufacturer's protocol. The primer sequences are summarised in Table 2. Real time RT-PCR was performed with a SYBR Green PCR Kit (TaKaRa, Japan), and the reactions were conducted on a RotorGene 3000A PCR instrument (Corbett, Australia). The housekeeping gene GAPDH was used as an internal control, and gene-specific mRNA expression was normalised against GAPDH expression.

\section{Western blot analysis}

The samples were homogenised in six volumes of lysis buffer containing $50 \mathrm{mmol} / \mathrm{L}$ Tris- $\mathrm{HCl}$ ( $\mathrm{pH} 7.5), 150 \mathrm{mmol} / \mathrm{L}$ $\mathrm{NaCl}, 12 \mathrm{mmol} / \mathrm{L} \mathrm{C}_{24} \mathrm{H}_{40} \mathrm{O}_{4} \cdot \mathrm{Na}, 0.1 \%$ SDS, $1 \mathrm{mmol} / \mathrm{L}$ EDTA, 1 $\mathrm{mmol} / \mathrm{L} \mathrm{MgCl}_{2}, 1 \mathrm{mmol} / \mathrm{L}$ phenylmethylsulphonyl fluoride (PMSF) and $2 \mu \mathrm{g} / \mathrm{mL}$ leupeptin. The protein concentration of the supernatant was determined using the Coomassie blue assay. The samples were electrophoresed by SDS-PAGE on $12 \%$ gels, transferred to polyvinylidene difluoride (PVDF) filters (Millipore, USA) and incubated with mouse anti-PAI-1 (BD, USA), rabbit anti-a-SMA (Epitomics, USA), rabbit antiAKT and anti-ERK (Bioworld, USA), rabbit anti-p-AKT and anti-p-ERK (Cell signal, USA), or rabbit anti- $\beta$-actin (Santa Cruz) antibodies. The primary antibodies were detected with horseradish peroxidase-conjugated second antibodies against mouse or rabbit IgG and an ECL luminescence system. The integral optical density (IOD) of each band was measured using a gel-image analysis system (Alpha Image 2200, Alpha, USA). The changes in the relative expression levels of PAI-1, a-SMA p-AKT, and p-ERK were represented by the ratio of each band's IOD to that of $\beta$-actin.

\section{Assay for the hydroxyproline content of lung tissue}

Wet lung samples weighing 30-100 mg were subjected to hydrolysis to determine the content of hydroxyproline according to the protocol of a Hydroxyproline Testing Kit (Jiancheng, Nanjing, China).

\section{Statistical analysis}

Statistical analysis of all reported values was performed with SAS8.0 software. The data were presented as the mean \pm SD and tested using one-way ANOVA for 4 groups on d 7, 14, and 28. $P$ values $<0.05$ were considered significant.

\section{Results}

PAI-1 expression increased in BLM-induced rat lung fibrosis and PAI-1 siRNA inhibited PAI-1 expression efficiently

First, we examined whether the PAI-1 expression was increased in BLM-induced rat lung fibrosis. Real time RT-PCR and Western blot analysis (Figure 1A, 1B) showed that the PAI-1 mRNA and protein levels in the lung were significantly increased in the BLM group compared with the sham group, and PAI-1 activity was significantly increased on $d 7,14$, and 28 in the BAL fluid (Figure 1C).

Second, the inhibition efficiency of PAI-1 expression was determined by intratracheal injection of PAI-1 siRNA into BLM-induced fibrotic lung tissue. Figure $1 \mathrm{~A}$ and $1 \mathrm{~B}$ show that compared with the BLM group, the expression levels of PAI-1 mRNA and protein were reduced significantly after intratracheal injection of the PAI-1 siRNA on $\mathrm{d} 7,14$, and 28 , whereas non-specific siRNA had no significant effect on PAI-1 mRNA and protein expression compared with the BLM group. In addition, the inhibitory effect gradually increased after the administration of the siRNA, corresponding to reductions of $29 \% \pm 9 \%, 58 \% \pm 9 \%$, and $67 \% \pm 7 \%$ in the PAI- 1 mRNA level and $46 \% \pm 11 \%, 51.9 \% \pm 5.3 \%$, and $65.5 \% \pm 4 \%$ in the PAI- 1 protein level at days 7, 14, and 28, respectively, compared with the BLM group. Figure $1 \mathrm{C}$ shows that administration of PAI-1 siRNA significantly decreased the level of PAI-1 in the BAL fluid compared with the BLM group, while the non-specific siRNA had no effect. The results indicated that the PAI-1 siRNA administered by intratracheal injection was delivered to the fibrotic area and silenced the expression of PAI-1 in BLM-induced lung fibrosis.

\section{PAI-1 siRNA ameliorated BLM-induced lung fibrosis in rats Changes in lung histological structure and collagen deposition}

To determine the effect of PAI-1 siRNA on lung fibrosis, we observed the histopathological changes and investigated the expression of pro-fibrogenic molecules. HE staining (Figure 2A) showed that the structure of the alveoli was greatly distorted in the BLM group. There was clear alveolitis represented by the infiltration of the pulmonary mesenchyme by neutrophils, fibroblasts, and macrophages on $\mathrm{d}$ 7. The infiltration of the cells gradually decreased on d 14 and 28. Masson's trichrome staining (Figure $2 \mathrm{~B}$ and $2 \mathrm{C}$ ) showed that there were small-sized collagen depositions on d 14, and large-scale of collagen accumulations were observed on d 28. In contrast, administration of PAI-1 siRNA alleviated the alveolitis significantly on $\mathrm{d} 7$ and suppressed the collagen deposition on $\mathrm{d}$ 14 and 28. The administration of non-specific siRNA had no effect compared with the BLM group.

\section{Changes in hydroxyproline content}

The content of hydroxyproline was measured to indicate collagen accumulation within the fibrotic lung tissues. The hydroxyproline content in lungs from rats with BLM-induced 

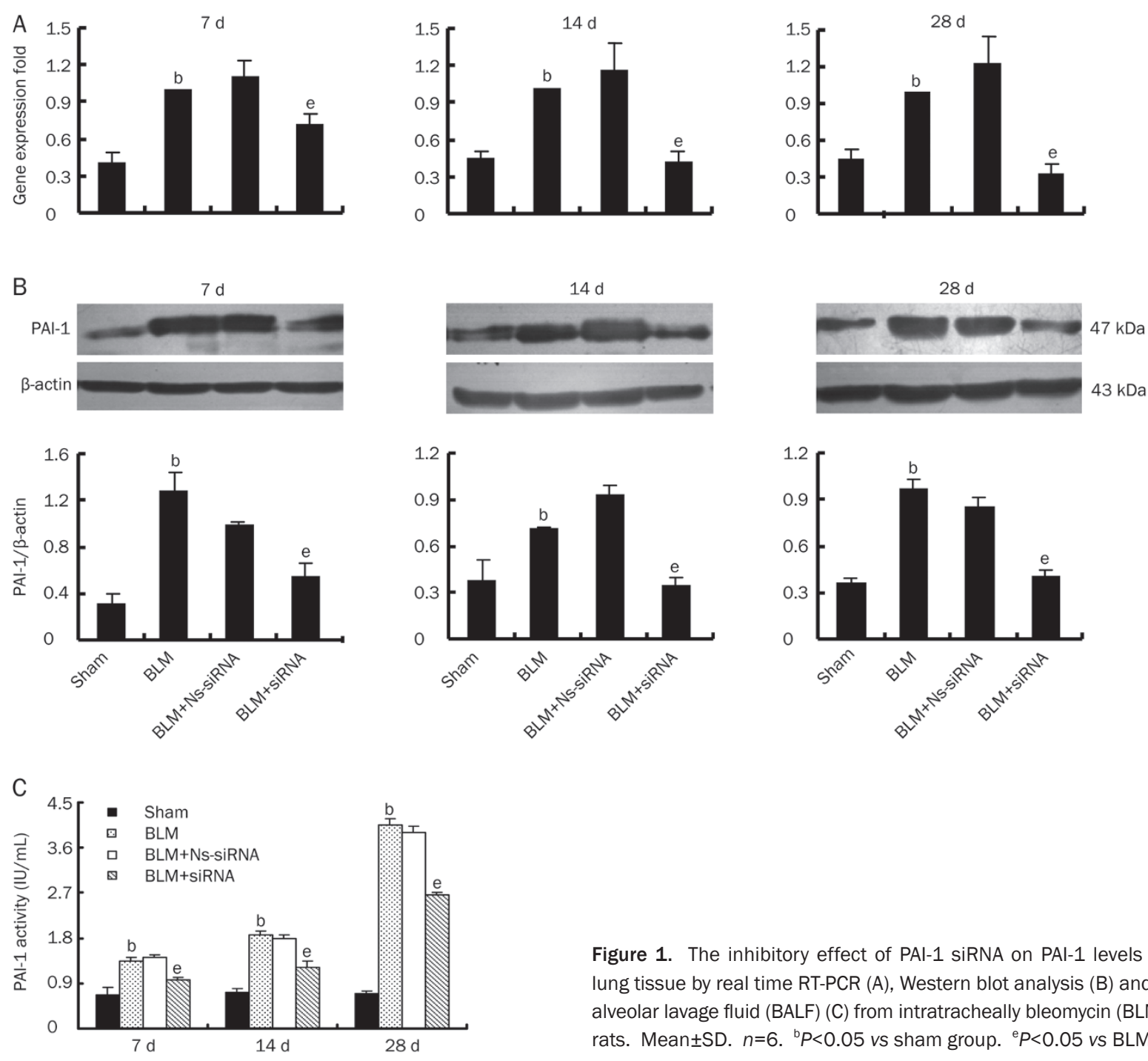

Figure 1. The inhibitory effect of PAl-1 siRNA on PAl-1 levels in fibrotic lung tissue by real time RT-PCR (A), Western blot analysis (B) and bronchoalveolar lavage fluid (BALF) (C) from intratracheally bleomycin (BLM)-treated rats. Mean \pm SD. $n=6$. ${ }^{b} P<0.05$ vs sham group. ${ }^{e} P<0.05$ vs BLM group.

lung fibrosis began to increase after $7 \mathrm{~d}$ and then increased rapidly thereafter. In the PAI-1 siRNA group, the hydroxyproline content was significantly decreased compared with that in the BLM group on d 14 and 28, while the non-specific siRNA had no effect on the hydroxyproline content compared with the BLM group (Figure 2D).

\section{Changes in $\alpha$-SMA expression}

Immunohistochemistry staining (Figure 3A) showed that pro-fibrogenic molecules in a-SMA-positive cells in the BLM model rats were mainly located in the endochylema and cytomembrane of myofibroblasts around the fibrotic areas, and their levels were significantly increased compared with the sham group. After treatment with the PAI-1 siRNA, the levels were significantly decreased on $\mathrm{d} 7,14$, and 28 . Western blot analysis (Figure 3B) showed that the protein level of a-SMA was significantly upregulated in the group with BLMinduced lung fibrosis compared with the sham group and that the upregulation was significantly inhibited on $d 7,14$, and
28 after treatment with PAI-1-targeted siRNA. Non-specific siRNA had no significant effect on the expression of a-SMA compared with the BLM group.

\section{Changes in collagen type-I and type-III}

Immunohistochemistry staining revealed that levels of collagen type-I (Figure 4A) and type-III (Figure 5A) were mainly located in the fibroblasts, myofibroblasts and mesenchyma of the fibrotic areas, and their expression levels increased significantly in the BLM group. After administration of siRNA, the levels significantly decreased. Real time RT-PCR analysis showed that the expression of collagen type-I and type-III expression were significantly increased in the lungs of BLMtreated rat, and the upregulation was significantly inhibited by the administration of PAI- 1 siRNA on $\mathrm{d} 7$ and 14 for collagen type-I (Figure 4B) and on d 14 and 28 for collagen typeIII (Figure 5B) compared with the BLM groups. Non-specific siRNA had no significant effects on the expression of collagen compared with the BLM group. 
A
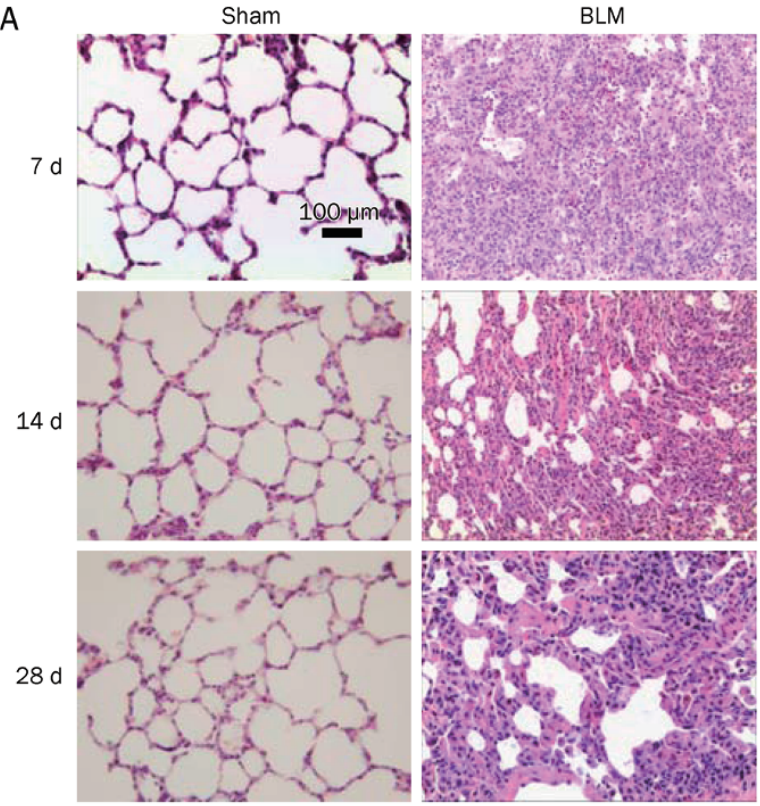

BLM+Ns-SiRNA
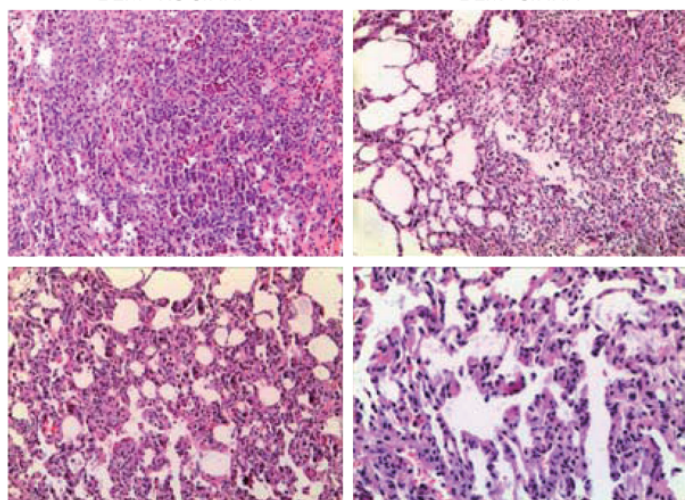
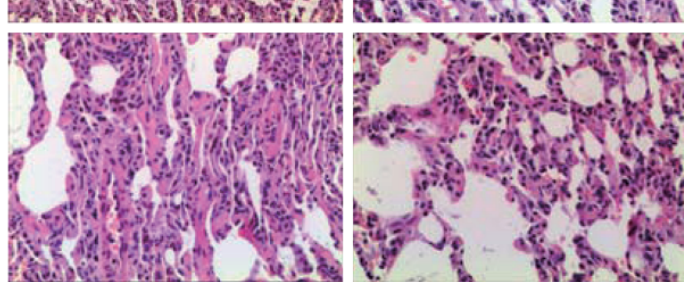

B
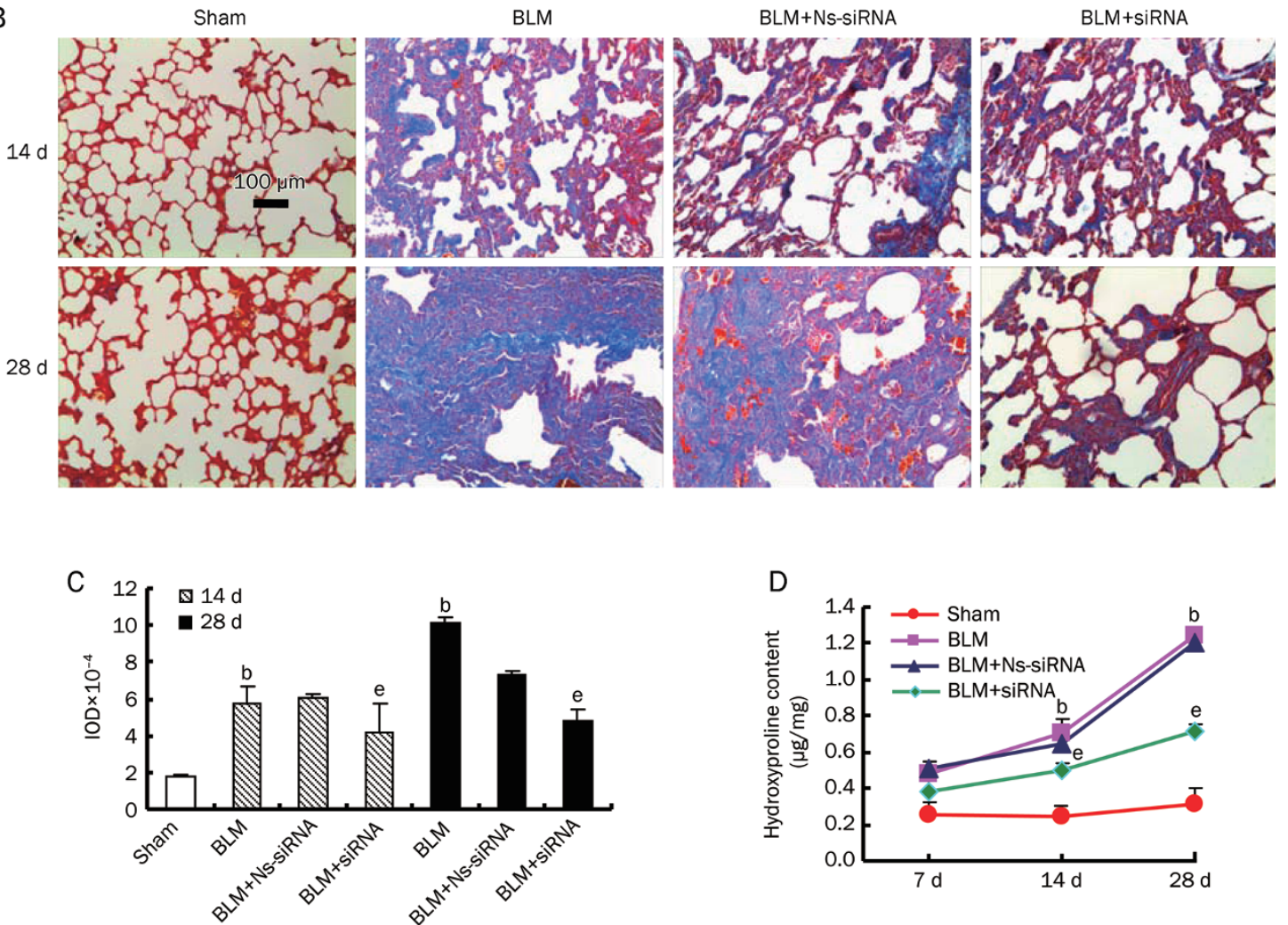

Figure 2. The effect of PAl-1 siRNA on intratracheally BLM-induced lung fibrosis. HE staining (A) and Masson' trichrome staining (B) shows the histological changes after intratracheal administration of PAI-1 siRNA. Scale bar in the upper-left microphotograph represents $100 \mu \mathrm{m}$, and this scale was used for all microphotographs. The bar graph in panel $\mathrm{C}$ is a quantitative representation of the changes in collagen based on the integral optical density (IOD). Mean \pm SD. $n=6$. ${ }^{\mathrm{b}} P<0.05$ vs sham group. ${ }^{\mathrm{e}} P<0.05$ vs BLM group. The line graph in panel $\mathrm{D}$ shows that the administration PAl-1 siRNA significantly inhibited the increase of hydroxyproline content in the fibrotic lung tissue induced by BLM treatment at the observed time points, whereas non-specific siRNA had no effect. 
A
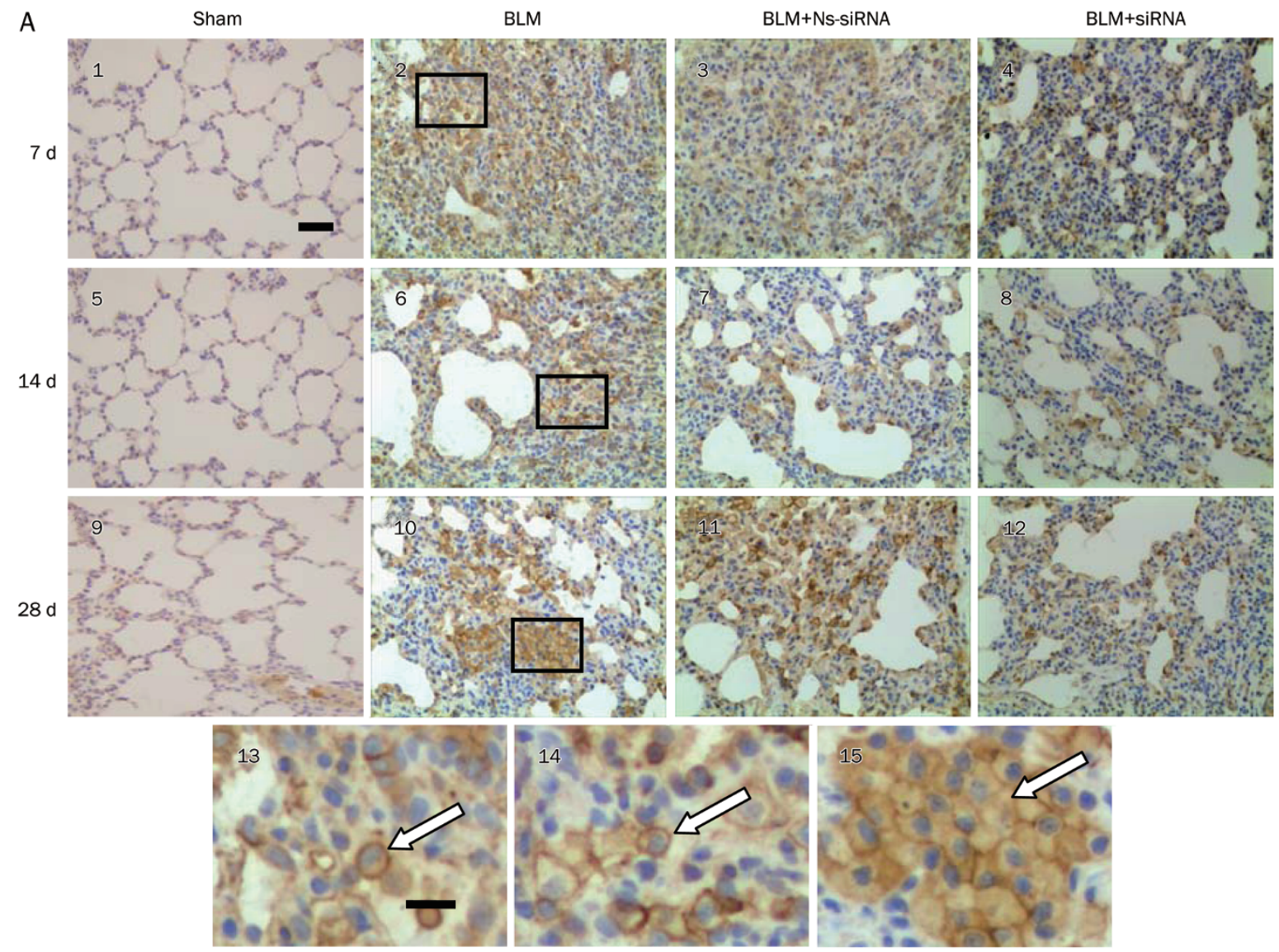

B
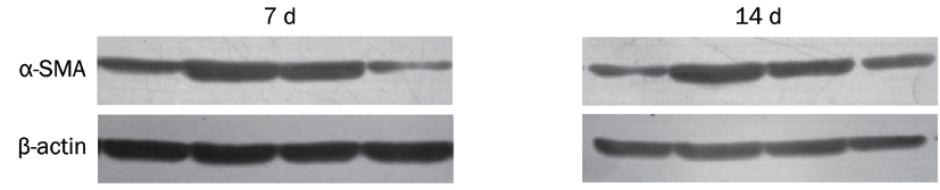

$28 d$
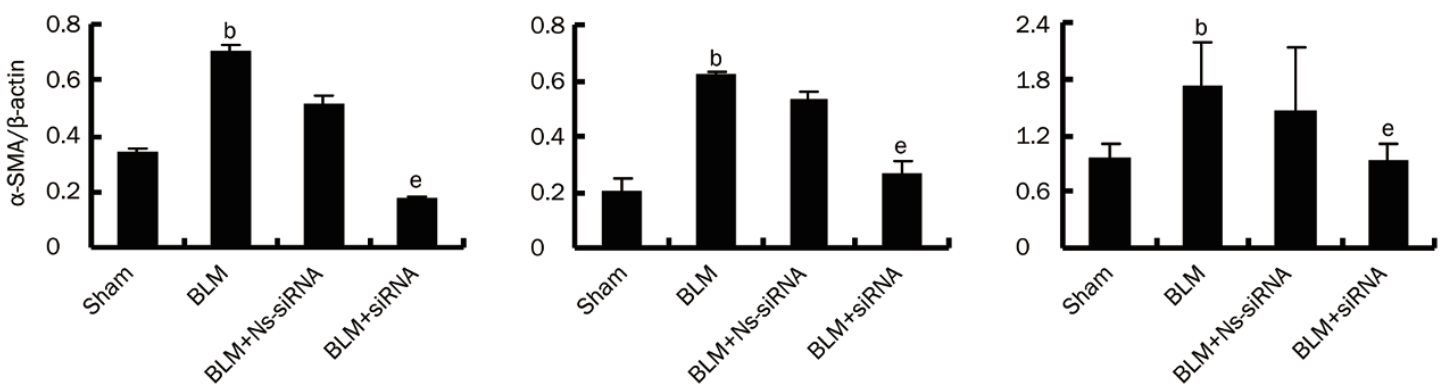

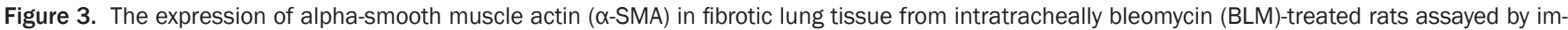

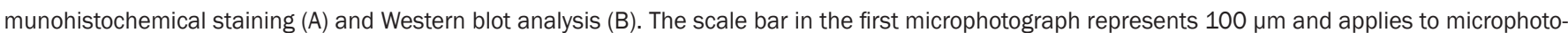

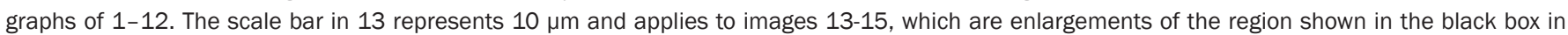
microphotographs 2, 6 and 10, respectively. Mean \pm SD. $n=6$. ${ }^{b} P<0.05$ vs sham group. ${ }^{e} P<0.05$ vs $B L M$ group.

Promoting apoptosis and inhibiting proliferation of pulmonary fibroblasts through the ERK and AKT pathways contributed to the anti-fibrosis effect of PAI-1 siRNA

\section{Changes in apoptosis and proliferation of fibroblasts}

To confirm the mechanism by which PAI-1 siRNA prevents lung fibrosis, we investigated whether down- or upregula- tion PAI-1 expression could affect proliferation and apoptosis in cultured rat fibroblasts. Figure 6A showed that the cells in which vimentin was positively expressed and a-SMA was negatively expressed were verified to be fibroblasts. Figure $6 \mathrm{~B}$ showed that the plasmid pcDNA-PAI-1 upregulated PAI-1 expression in fibroblasts by $277 \%$ and $204 \%$ at $48 \mathrm{~h}$ and $72 \mathrm{~h}$, 
A
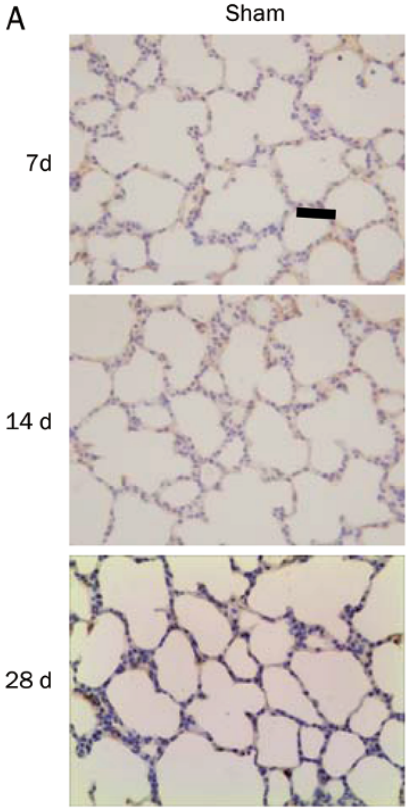

BLM

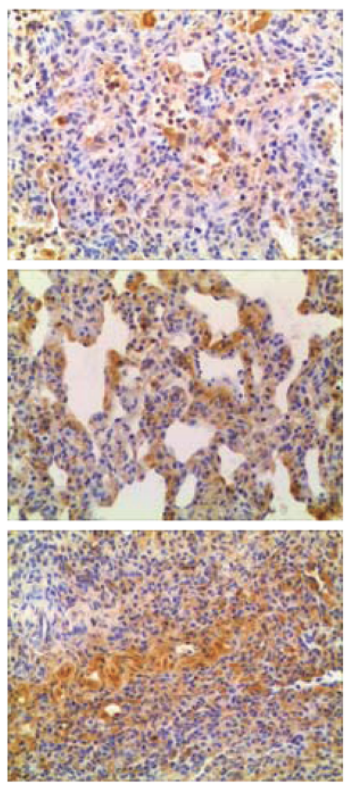

BLM+Ns-siRNA

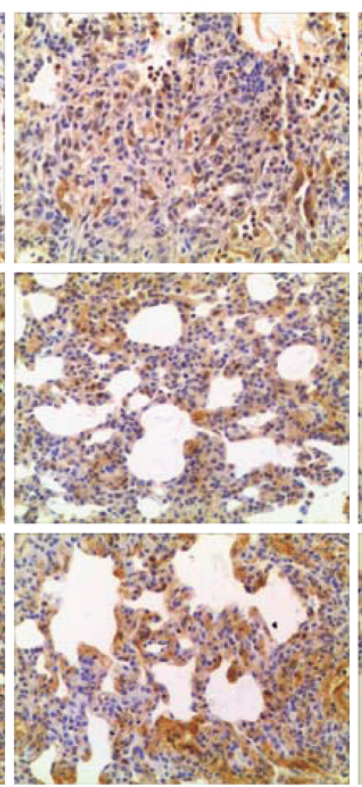

BLM+siRNA

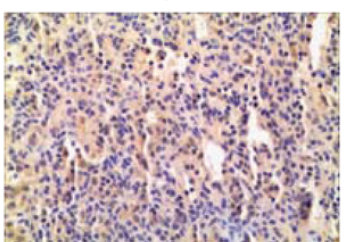

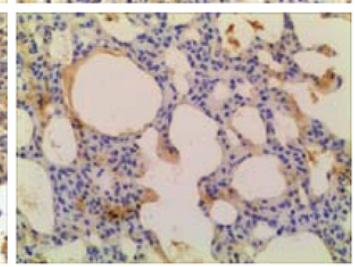
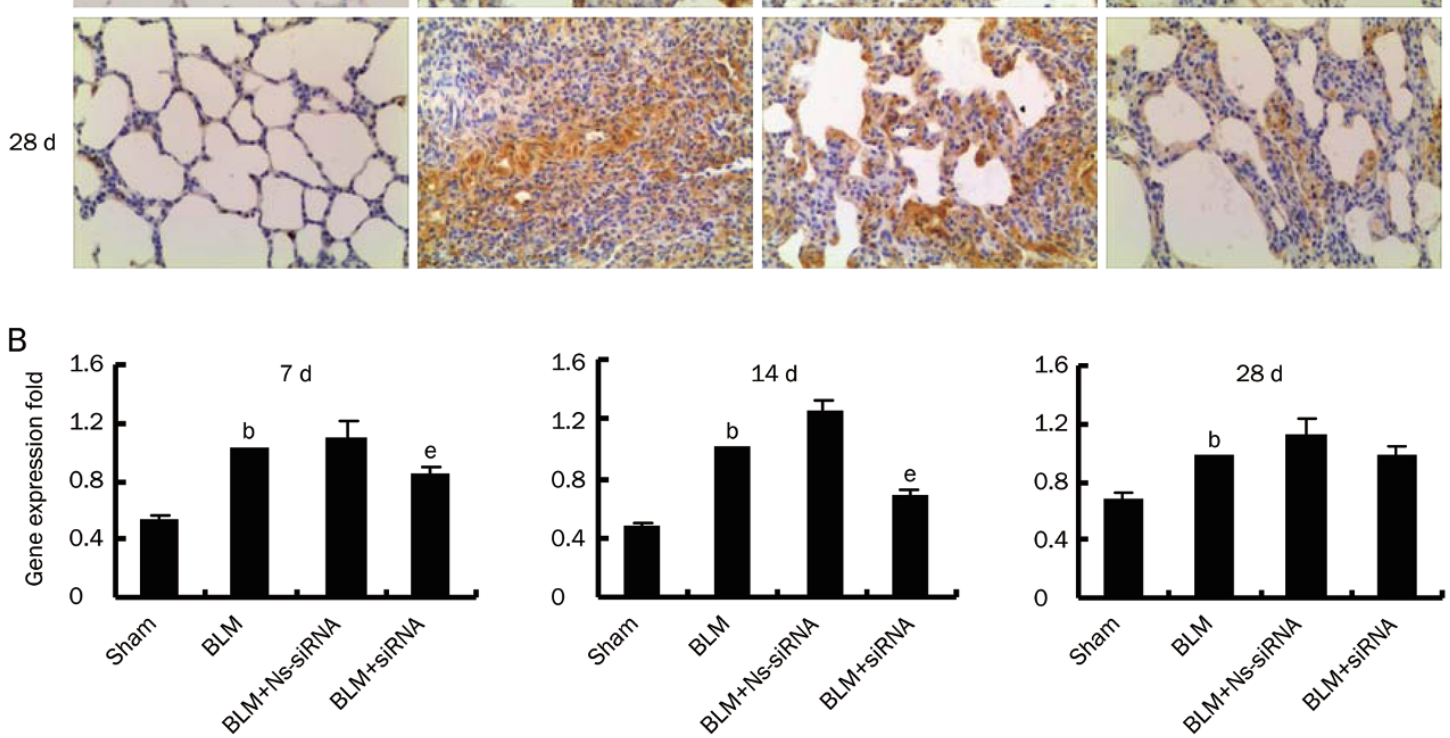

Figure 4. The expression of collagen type-l in the fibrotic lung tissue of the intratracheally bleomycin (BLM)-treated rats as assayed by immunohistochemical staining (A) and real time RT-PCR (B). Scale bar in the upper-left microphotograph represents $100 \mu \mathrm{m}$ and applies to all microphotographs. Mean \pm SD. $n=6$. ${ }^{\mathrm{b}} P<0.05$ vs sham group. ${ }^{e} P<0.05$ vs BLM group.

respectively. Figure $6 \mathrm{C}$ showed that the proliferation of fibroblasts in the siRNA group was significantly inhibited, and the inhibitory effect peaked at $48 \mathrm{~h}$ and descended $72 \mathrm{~h}$ after the transfection of PAI-1 siRNA. However, the proliferation of fibroblasts was significantly stimulated until $72 \mathrm{~h}$ after transfection of pcDNA-PAI-1, according to the results of the MTT assay.

Apoptosis was evaluated by detecting the levels of caspase-3. Real time RT-PCR analysis (Figure 6D) shows that caspase-3 expression was upregulated after transfection of PAI-1 siRNA, whereas the expression was downregulated after transfection of pcDNA-PAI-1 into fibroblasts. In vivo (Figure $6 \mathrm{E})$, immunohistochemistry staining showed that levels of caspase-3 increased after intratracheal treatment of BLM-induced lung tissue with PAI-1 siRNA. These results indicated that PAI-1 siRNA ameliorated lung fibrosis directly by inhibiting the proliferation and promoting the apoptosis of fibroblasts.

\section{Changes in expression of $p-E R K 1 / 2$ and $p-A K T$}

To investigate the role of the ERK and AKT signaling path- ways in the amelioration of lung fibrosis by PAI-1 siRNA, we analysed the protein expression of phosphorylated-AKT and phosphorylated-ERK1/2 in fibrotic lung tissue because the level of phosphorylation represents the activation of these signalling pathways. As shown in Figure 7, the expression of p-AKT and p-ERK significantly increased in BLM-treated rat lung tissues on $\mathrm{d} 7,14$, and 28, and the expression levels were downregulated at the observed time points after intratracheal treatment with PAI-1 siRNA compared with the BLM group. The ERK and AKT signaling pathways contributed to the antipulmonary fibrosis effect of PAI-1 siRNA.

\section{Discussion}

Local delivery of siRNA to the lungs, for example, through inhalation or intranasal administration, offers a unique opportunity to treat respiratory diseases, including inflammation, cystic fibrosis, infectious diseases, and cancer ${ }^{[11]}$. The effectiveness of in vivo gene knockdown has been a major question among many investigators ${ }^{[12]}$. In the present study, to improve the effectiveness of siRNA, repeat intratracheal injection twice 
A
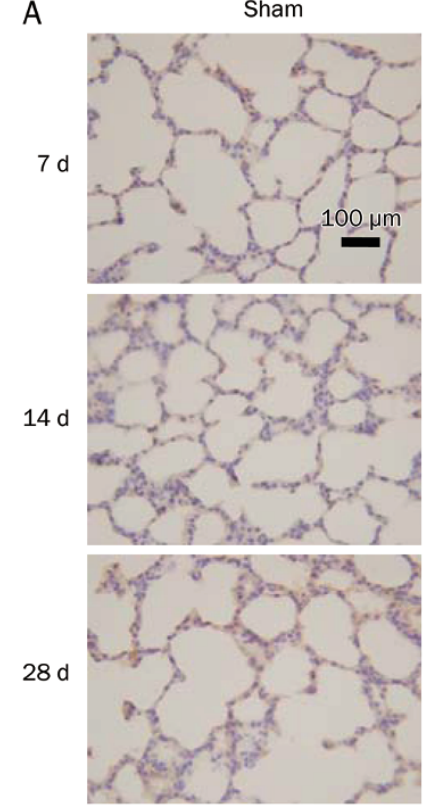

B

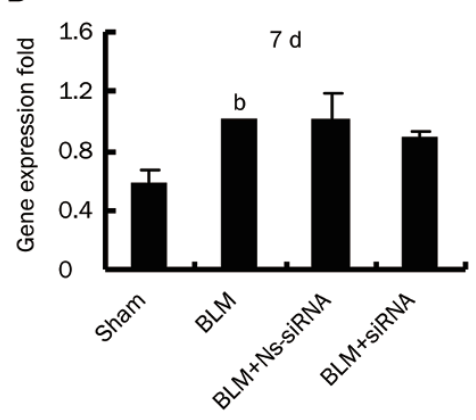

BLM
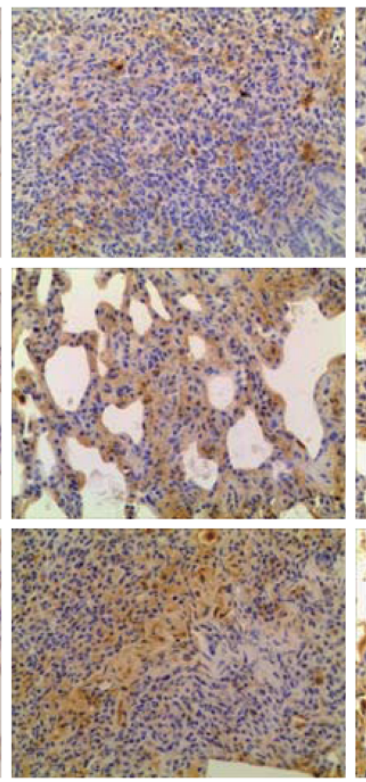

BLM+Ns-siRNA

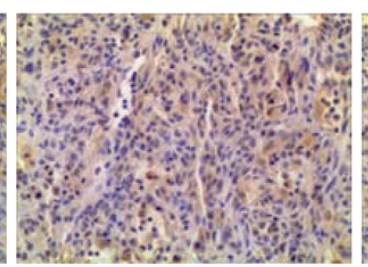

BLM-siRNA
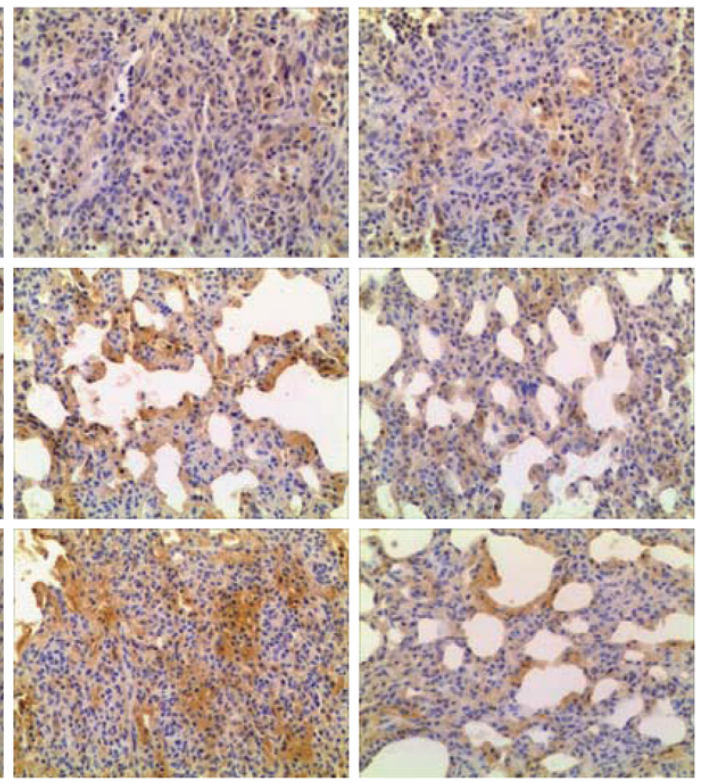
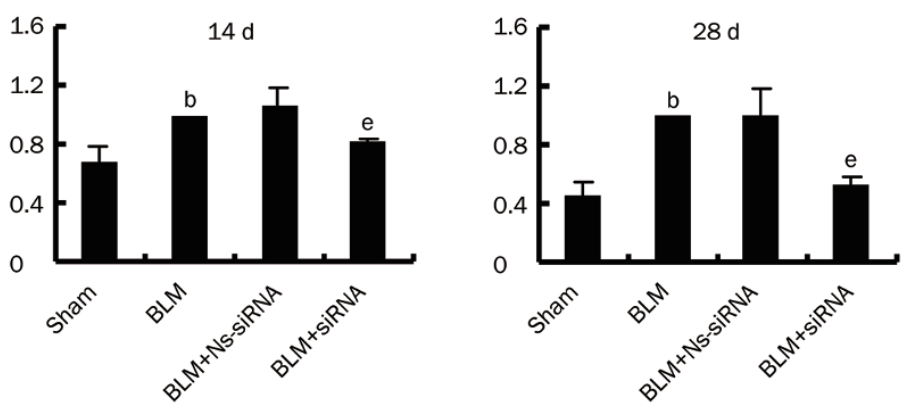

Figure 5. The expression of collagen type-III in the fibrotic lung tissue of the intratracheally bleomycin (BLM)-treated rats as assayed by immunohistochemical staining (A) and real time RT-PCR (B). Mean \pm SD. $n=6$. ${ }^{b} P<0.05$ vs sham group. ${ }^{e} P<0.05$ vs BLM group.

a week beginning during the alveolitis phase and extending through the fibrotic phase was performed in a BLM-induced model of rat lung injury. Intriguingly, the repeated administration of PAI-1 siRNA markedly downregulated PAI-1 gene and protein expression as well as the PAI-1 level in the BAL fluid. These findings indicate that repeated intratracheal injection is a highly effective approach for siRNA delivery to reduce PAI-1 levels.

Our study demonstrated that the expression of PAI-1 was increased in rats with BLM-induced lung fibrosis, and the PAI-1 activity in the BAL fluid increased gradually along with the exacerbation of lung fibrosis. Intriguingly, several studies have confirmed that PAI-1 was an important factor during the transformation of fibroblasts to myofibroblasts, which amplified the inflammatory response and resulted in the progression of fibrosis ${ }^{[13-15]}$. Offersen et al observed that myofibroblasts were the predominant PAI-1-expressing cell in breast carcinomas based on immunohistochemistry double staining ${ }^{[16]}$. Another study showed that the crucial step was the cleavage of the uPA receptor to D2D3 after binding of PAI-1 to uPA and the uPA receptor during fibroblast-to-myofibroblast differentiation ${ }^{[17]}$. Hu et al observed that hepatic fibrosis in rats could be ameliorated by downregulating the expression of PAI- ${ }^{[18]}$. Furthermore, Senoo et al reported that intranasal administration of PAI-1 siRNA attenuated the progression of pulmonary fibrosis ${ }^{[10]}$ and therefore suggested that PAI-1 might be an important factor capable of accelerating lung fibrosis. The present study provided additional direct evidence to support this assumption by observing the expression of PAI-1 in the fibrotic pulmonary tissue of BLM-treated rats. We found that intratracheal injection of PAI-1 siRNA significantly reduced the level of PAI-1 in the fibrotic pulmonary tissue of BLMtreated rats, and this suppression was accompanied by the amelioration of alveolitis, the inhibition of the transformation of fibroblasts through downregulation of the expression of a-SMA, and a reduction in the synthesis of collagen in fibrotic lung. These findings convincingly indicated that PAI-1 played an important role in the pathogenesis of BLM-induced lung fibrosis and that inhibition of PAI-1 expression with siRNA might be a potential therapeutic target for treatment of lung 
A

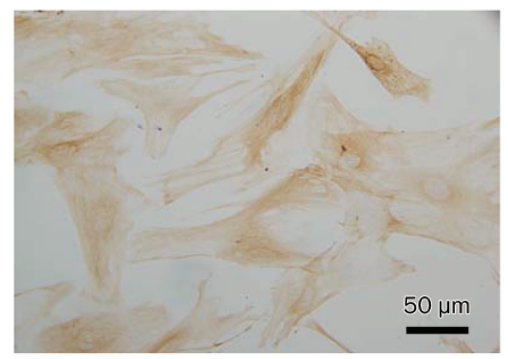

C

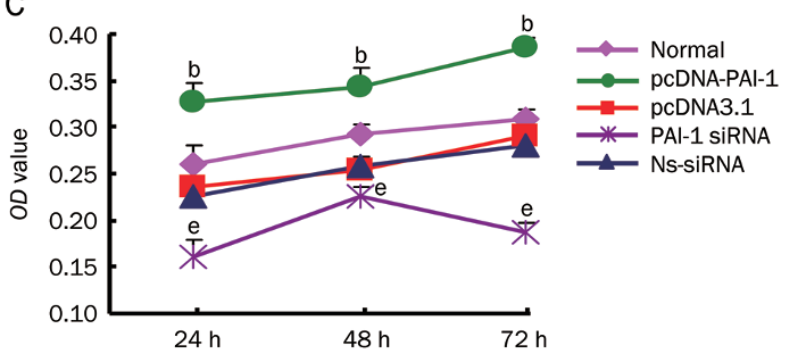

B
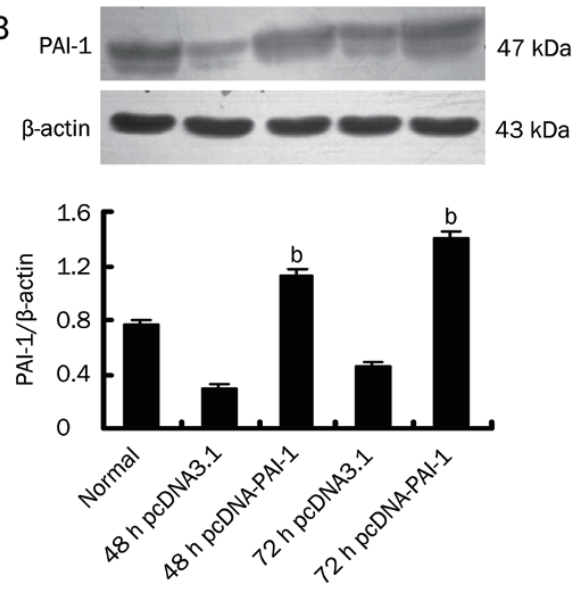

D

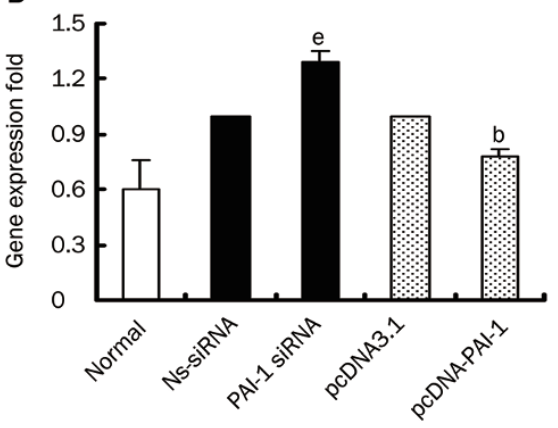

E
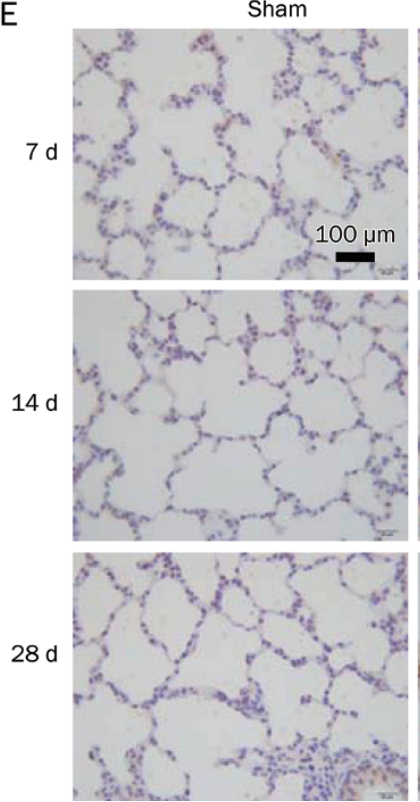

BLM
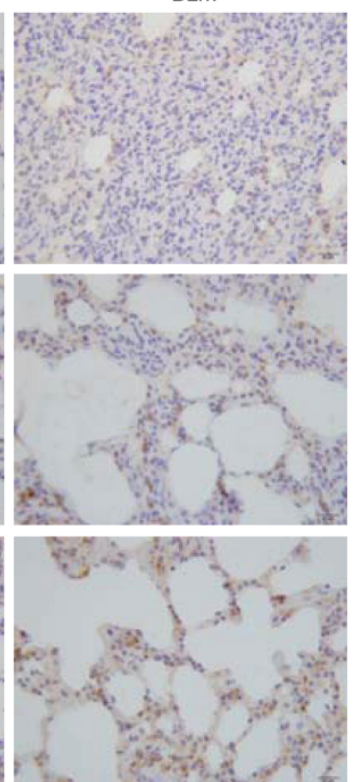

BLM+Ns-SiRNA
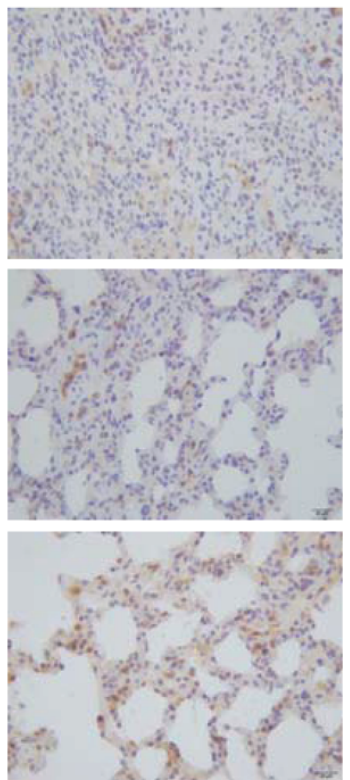

BLM+siRNA
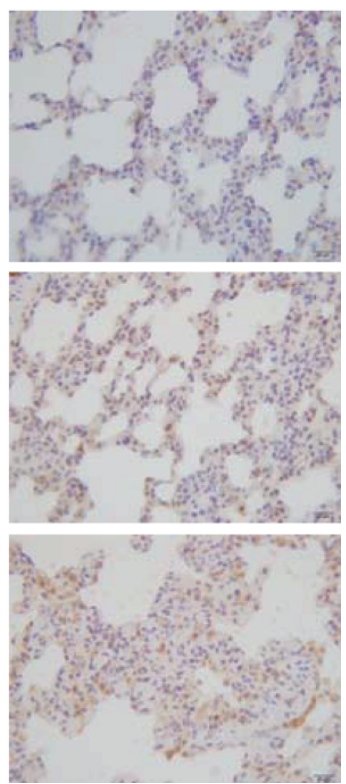

Figure 6. The effects of regulating PAl-1 expression on the proliferation and apoptosis of rat fibroblasts. Immunohistochemistry (A) shows that Vimentin was positively expressed, confirming that these cells are fibroblasts. The scale bar represents $50 \mu \mathrm{m}$. Western blot analysis (B) shows the effect of plasmid pcDNA-PAI-1 on the expression of PAI-1 in fibroblasts. The line graph in panel (C) shows the effect on proliferation after down- or up-regulation of PAI-1 expression based on the MTT assay. Real time RT-PCR analysis (D) shows the caspase-3 expression after transfection with PAI-1 siRNA and pcDNA-PAI-1. Immunohistochemistry (E) shows the caspase-3 expression after intratracheal introduction of PAI-1 siRNA into BLM-induced lung tissue. The scale bar represents $100 \mu \mathrm{m}$. Mean \pm SD. $n=6$. ${ }^{b} P<0.05$ vs pcDNA3.1. ${ }^{e} P<0.05$ vs Ns-siRNA. 
A
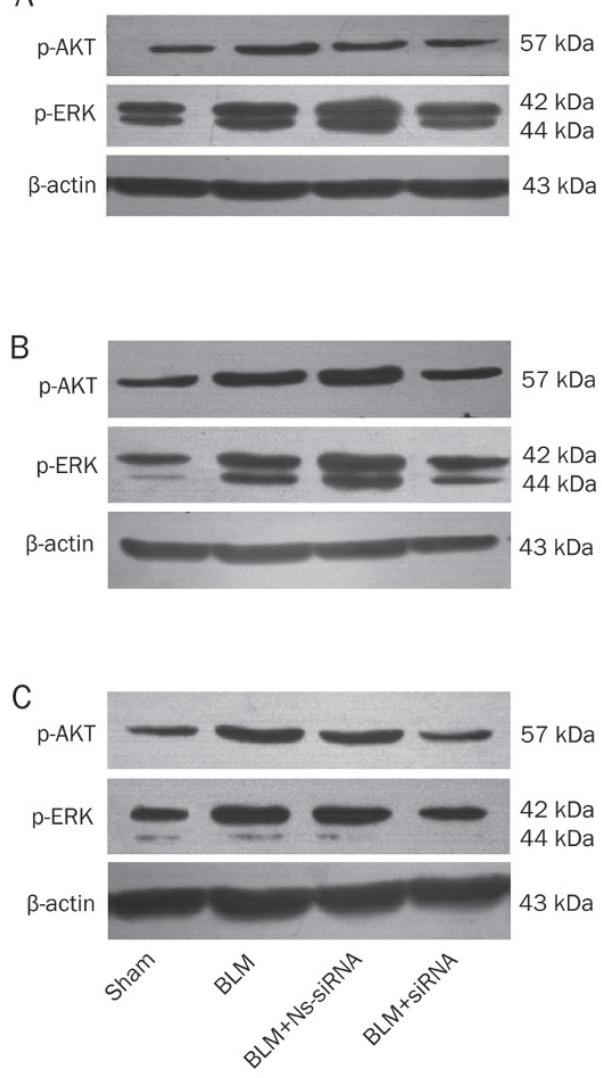
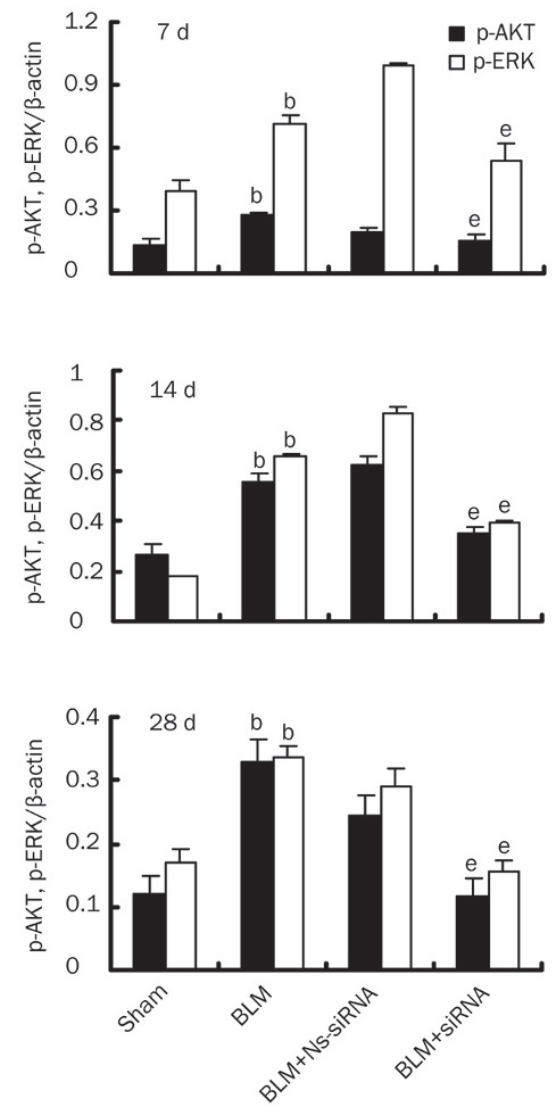

Figure 7. Western blot analysis shows the expression of phosphorylated-extracellular signal-regulated kinase 1/2 (p-ERK1/2) and phosphatidylinositol 3-phosphate kinase $(\mathrm{PI} K \mathrm{~K}) /$ Akt ( $\mathrm{p}-\mathrm{AKT}$ ) in fibrotic lung tissue from bleomycin (BLM)-treated rats. The left panels are representative bands from a Western blotting, and the right panels show quantitative representations of the integral optical density (IOD) of the bands. The ratio between the IOD for the band of each target protein and that for $\beta$-actin is used for the statistical analysis. ${ }^{\mathrm{b}} P<0.05$ vs sham group, and ${ }^{\mathrm{e}} P<0.05$ vs BLM group. fibrosis in a clinical setting.

PAI-1 regulates a variety of cellular processes, including adhesion, migration, detachment, angiogenesis, proliferation and apoptosis, in vitro and in vivo ${ }^{[19,20]}$. PAI-1 is a paradoxical protein that exerts different effects in various cell lines ${ }^{[21-24]}$. Previous studies have shown that PAI-1 could protect fibrosarcoma cells from etoposide-induced apoptosis ${ }^{[25]}$ and augment the proliferation of vascular smooth muscle cells ${ }^{[26]}$. To explore the mechanisms by which PAI-1 siRNA prevents lung fibrosis, we observed the effects of transfection of PAI-1 siRNA and pcDNA-PAI-1 on the proliferation and apoptosis of lung fibroblasts. In vitro, we found that downregulation of PAI-1 expression by PAI-1 siRNA could inhibit proliferation and promote the expression of caspase- 3 of fibroblasts, whereas upregulation of PAI-1 expression by pcDNA-PAI-1 could promote fibroblast proliferation and inhibit the expression of caspase-3. These results revealed that PAI-1 siRNA inhibited lung fibrosis by promoting apoptosis by determining caspase- 3 and suppressing the proliferation of fibroblasts. These findings represent new insights into the mechanisms underlying the anti-fibrotic effects of PAI-1 siRNA in the lungs. In a previous study, Senoo et al revealed that PAI-1 siRNA can inhibit lung fibrosis by inhibiting EMT induced by TGF- $\beta$ in LA-4 cells ${ }^{[18]}$. Bauman et al observed that plasminogen activation promoted the release of hepatocyte growth factor to promote prostaglandin $\mathrm{E}_{2}\left(\mathrm{PGE}_{2}\right)$ synthesis and fibroblast apopto$\operatorname{sis}^{[27]}$.
It is generally accepted that PAI-1 binding to uPA and the $\mathrm{uPA}$ receptor induces internalisation of the complex, thereby facilitating extracellular proteolysis as well as activation of growth factors and anti-apoptosis proteins ${ }^{[28,29]}$. Furthermore, these intermediates activate several signalling pathways. For example, the uPA-uPAR-PAI-1 complex interacts with integrins to induce focal adhesion kinase (FAK) and ERK activation $^{[30]}$ via low-density lipoprotein receptor-related protein (LRP-1). This complex also regulates ras-related C3 botulinum toxin substrate 1 (Rac1) and ERK activation ${ }^{[31]}$, the JAK-STAT signalling pathway, the ERK/MAPK and PI3K/AKT pathways $^{[32]}$, and it modulates the AKT pathway in wild-type and PAI-1-deficient endothelial cells ${ }^{[33]}$. Therefore, it is possible that the ERK and AKT signalling pathways play an important role in IPF. Zhong et al demonstrated that selective inhibition of ERK1 by AdshERK1 could significantly attenuate the deposition of the extracellular matrix in fibrotic liver models ${ }^{[34]}$. In the present study, we report that the expression of $\mathrm{p}-\mathrm{AKT}$ and p-ERK was significantly increased in a BLM-induced model of lung fibrosis, whereas the expression of p-AKT and p-ERK was significantly decreased after downregulation of the expression of PAI-1 by siRNA. These results suggested that inhibition of the $\mathrm{p}$-AKT and $\mathrm{p}$-ERK protein levels may participate in the inhibition of proliferation and the stimulation of apoptosis observed in fibroblasts treated with PAI-1 siRNA. These results also suggested that the PI3K/AKT signaling pathway and the ERK-signalling pathway were involved in 
IPF progression. This is another novel finding of the present study that has not been previously reported. Further investigation should be carried out regarding whether downregulation of the expression of ERK and AKT proteins ameliorates lung fibrosis.

In summary, our results indicate the existence of a significant functional role of PAI-1 in the development of lung fibrosis and suggest that PAI-1 is a potential target that is worth exploiting in the treatment of IPF by using PAI-1-targeted siRNA. In addition, we provide the first strong evidence that suppression of the levels of p-AKT and p-ERK in fibrotic lung tissue by PAI-1-targeted siRNA is associated with the suppression of fibroblast proliferation.

\section{Acknowledgements}

We gratefully acknowledge Dr Fang WANG for providing the plasmid pcDNA-PAI-1 and the vector pcDNA3.1; Dr Wenxin WU and Cui-qing MA for their technical assistance; and Madam Li-mei LIU for her assistance with the writing.

This work was funded by the National Natural Science Foundation of China (No 30770738; No 31040036 ; No 81000477), the Key Base Research Project of Hebei Province (№ 11966121D), and the Natural Science Foundation of Hebei Province (№ C2009001161).

\section{Author contribution}

Yan-ping ZHANG and Wen-bin LI were responsible for the conception and design of the research. Yan-ping ZHANG, Wei-li WANG, and Jian LIU performed the experiments; Weili WANG and Lin-lin BAI analysed the data; Ya-dong YUAN and Min ZHANG interpreted the results of the experiments; Yu-yan HU prepared the figures; Yan-ping ZHANG drafted the manuscript; Wen-bin LI and Shu-xia SONG revised the manuscript; Yan-ping ZHANG and Wen-bin LI approved the final version of the manuscript;

\section{References}

1 Doubková M, Skhicková J. Idiopathic pulmonary fibrosis. Vnitr Lek 2005; 51: 1375-84.

2 Hinz B, Phan SH, Thannickal VJ, Galli A, Bochaton-Piallat ML, Gabbiani G. The myofibroblast: one function, multiple origins. Am J Pathol 2007; 170: 1807-16.

3 Hildenbrand R, Gandhari M, Stroebel P, Marx A, Allgayer H, Arens N. The urokinase-system-role of cell proliferation and apoptosis. Histol Histopathol 2008; 23: 227-36.

4 Bueno M, Salgado S, Beas-Zárate C, Armendariz-Borunda J. Urokinase-type plasminogen activator gene therapy in liver cirrhosis is mediated by collagens gene expression down-regulation and upregulation of MMPs, HGF and VEGF. J Gene Med 2006; 8: 1291-9.

5 Hattori N, Mizuno S, Yoshida Y, Chin K, Mishima M, Sisson TH, et al. The plasminogen activation system reduces fibrosis in the lung by a hepatocyte growth factor-dependent mechanism. Am J Pathol 2004; 164: 1091-8.

6 Bertozzi P, Astedt B, Zenzius L, Lynch K, LeMaire F, Zapol W, et al. Depressed bronchoalveolar urokinase activity in patients with adult respiratory distress syndrome. N Engl J Med 1990; 322: 890-7.

7 Eitzman DT, McCoy RD, Zheng X, Fay WP, Shen T, Ginsburg D, et al.
Bleomycin-induced pulmonary fibrosis in transgenic mice that either lack or overexpress the murine plasminogen activator inhibitor-1 gene. J Clin Invest 1996; 97: 232-7.

8 Chuang-Tsai S, Sisson TH, Hattori N, Tsai CG, Subbotina NM, Hanson $\mathrm{KE}$, et al. Reduction in fibrotic tissue formation in mice genetically deficient in plasminogen activator inhibitor-1. Am J Pathol 2003; 163: 445-52.

9 Hu PF, Zhu YW, Zhong W, Chen YX, Lin Y, Zhang X, et al. Inhibition of plasminogen activator inhibitor-1 expression by siRNA in rat hepatic stellate cells. J Gastroenterol Hepatol 2008; 23: 1917-25.

10 Senoo T, Hattori N, Tanimoto T, Furonaka M, Ishikawa N, Fujitaka $\mathrm{K}$, et al. Suppression of plasminogen activator inhibitor-1 by RNA interference attenuates pulmonary fibrosis. Thorax 2010; 65: 33440.

11 Durcan N, Murphy C, Cryan SA. Inhalable siRNA: potential as a therapeutic agent in the lungs. Mol Pharm 2008; 5: 559-66.

12 Castanotto D, Rossi1 JJ. The promises and pitfalls of RNAinterference-based therapeutics. Nature 2009; 457: 426-33.

13 Thannickal VJ, Horowitz JC. Evolving concepts of apoptosis in idiopathic pulmonary fibrosis. Proc Am Thorac Soc 2006; 3: 350-6.

14 Hinz B, Gabbiani G. Cell-matrix and cell-cell contacts of myofibroblasts: role in connective tissue remodeling. Thromb Haemost 2003; 90: 993-1002.

15 Phan SH. The myofibroblast in pulmonary fibrosis. Chest 2002; 122 : 286S-9S.

16 Offersen BV, Nielsen BS, Høyer-Hansen G, Rank F, Hamilton-Dutoit S, Overgaard J, et al. The myofibroblast is the predominant plasminogen activator inhibitor-1-expressing cell type in human breast carcinomas. Am J Pathol 2003; 163: 1887-99.

17 Bernstein AM, Twining SS, Warejcka DJ, Tall E, Masur SK. Urokinase receptor cleavage: a crucial step in fibroblast-to-myofibroblast differentiation. Mol Bio Cell 2007; 18: 2716-27.

18 Hu PF, Chen H, Zhong W, Lin Y, Zhang X, Chen YX, et al. Adenovirusmediated transfer of siRNA against PAl-1 mRNA ameliorates hepatic fibrosis in rats. J Hepatol 2009; 51: 102-13.

19 Dellas C, Loskutoff DJ. Historical analysis of PAl-1 from its discovery to its potential role in cell motility and disease. Thromb Haemost 2005; 93: 631-40.

20 Binder BR, Mihaly J, Prager GW. UPAR-uPA-PAI-1 interactions and signaling: a vascular biologist's view. Thromb Haemost 2007; 97: 336-42.

21 Balsara RD, Ploplis VA. Plasminogen activator inhibitor-1: the doubleedged sword in apoptosis. Thromb Haemost 2008; 100: 1029-36.

22 Lademann UA, Rømer MU. Regulation of programmed cell death by plasminogen activator inhibitor type 1 (PAl-1). Thromb Haemost 2008; 100: 1041-6.

23 Al-Fakhri N, Chavakis T, Schmidt-Wöll T, Huang B, Cherian SM, Bobryshev YV, et al. Induction of apoptosis in vascular cells by plasminogen activator inhibitor-1 and high molecular weight kininogen correlates with their anti-adhesive properties. Biol Chem 2003; 384: 423-35.

24 Chen SC, Henry DO, Reczek PR, Wong MK. Plasminogen activator inhibitor-1 inhibits prostate tumor growth through endothelial apoptosis. Mol Cancer Ther 2008; 7: 1227-36.

25 Rømer MU, Larsen L, Offenberg H, Brünner N, Lademann UA. Plasminogen activator inhibitor 1 protects fibrosarcoma cells from etoposide-induced apoptosis through activation of the PI3K/Akt cell survival pathway. Neoplasia 2008; 10: 1083-91.

26 Chen Y, Budd RC, Kelm RJ Jr, Sobel BE, Schneider DJ. Augmentation of proliferation of vascular smooth muscle cells by plasminogen activator inhibitor type 1. Arterioscler Thromb Vasc Biol 2006; 26: 
1777-83.

27 Bauman KA, Wettlaufer SH, Okunishi K, Vannella KM, Stoolman JS, Huang SK, et al. The antifibrotic effects of plasminogen activation occur via prostaglandin E2 synthesis in humans and mice. J Clin Invest 2010; 120: 1950-60.

28 Huai Q, Mazar AP, Kuo A, Parry GC, Shaw DE, Callahan J, et al. Structure of human urokinase plasminogen activator in complex with its receptor. Science 2006; 311: 656-9.

29 Horowitz JC, Rogers DS, Simon RH, Sisson TH, Thannickal VJ. Plasminogen activation induced pericellular fibronectin proteolysis promotes fibroblast apoptosis. Am J Respir Cell Mol Biol 2008; 38 : 78-87.

30 Aguirre-Ghiso JA, Estrada Y, Liu D, Ossowski L. ERK (MAPK) activity as a determinant of tumor growth and dormancy; regulation by p38 (SAPK). Cancer Res 2003; 63: 1684-95.
31 Ma Z, Thomas KS, Webb DJ, Moravec R, Salicioni AM, Mars WM, et al. Regulation of Rac1 activation by the low density lipoprotein receptorrelated protein. J Cell Biol 2002; 159: 1061-70.

32 Kiian I, Tkachuk N, Haller H, Dumler I. Urokinase-induced migration of human vascular smooth muscle cells requires coupling of the small GTPases RhoA and Rac1 to the Tyk2/PI3-K signalling pathway. Thromb Haemost 2003; 89: 904-14.

33 Balsara RD, Castellino FJ, Ploplis VA. A novel function of plasminogen activator inhibitor-1 in modulation of the AKT pathway in wild-type and plasminogen activator inhibitor-1-deficient endothelial cells. J Biol Chem 2006; 281: 22527-36.

34 Zhong W, Shen WF, Ning BF, Hu PF, Lin Y, Yue HY, et al. Inhibition of extracellular signal-regulated kinase 1 by adenovirus mediated small interfering RNA attenuates hepatic fibrosis in rats. Hepatology 2009; 50: $1524-36$. 